\title{
MEASURING BARYON ACOUSTIC OSCILLATIONS WITH MILLIONS OF SUPERNOVAE
}

\author{
Hu Zhan, ${ }^{1}$ Lifan Wang, ${ }^{2}$ Philip Pinto, ${ }^{3}$ And J. Anthony Tyson ${ }^{1}$ \\ Received 2007 September 10; accepted 2008 January 16; published 2008 February 4
}

\begin{abstract}
Since Type Ia supernovae ( $\mathrm{SNe}$ ) explode in galaxies, they can, in principle, be used as the same tracer of the large-scale structure as their hosts to measure baryon acoustic oscillations (BAOs). To realize this, one must obtain a dense integrated sampling of SNe over a large fraction of the sky, which may only be achievable photometrically with future projects such as the Large Synoptic Survey Telescope. The advantage of SN BAOs is that $\mathrm{SNe}$ have more uniform luminosities and more accurate photometric redshifts than galaxies, but the disadvantage is that they are transitory and hard to obtain in large number at high redshift. We find that a halfsky photometric SN survey to redshift $z=0.8$ is able to measure the baryon signature in the SN spatial power spectrum. Although dark energy constraints from SN BAOs are weak, they can significantly improve the results from SN luminosity distances of the same data, and the combination of the two is no longer sensitive to cosmic microwave background priors.
\end{abstract}

Subject headings: cosmological parameters — distance scale — large-scale structure of universe

\section{INTRODUCTION}

Type Ia supernovae ${ }^{4}(\mathrm{SNe})$ have become a mature tool for studying the cosmic expansion history (e.g., Phillips 1993; Riess et al. 1998; Perlmutter et al. 1999). A number of SN surveys, such as the Sloan Digital Sky Survey (SDSS) II (Frieman et al. 2008), the Supernova Legacy Survey (Astier et al. 2006), and the ESSENCE Supernova Survey (Miknaitis et al. 2007), are being carried out to improve the statistics and our understanding of the systematics. Moreover, the SN technique will be an integral part of almost every proposed dark energy survey including the Large Synoptic Survey Telescope ${ }^{5}$ (LSST; see Tyson 2006) and the Joint Dark Energy Mission.

The conventional SN technique, measuring only the relative luminosity distance, $D_{L}$, is subject to degeneracies between cosmological parameters. For example, the SN constraint on the dark energy equation-of-state (EOS, $w$ ) parameter $w_{a}$, as defined by $w(z)=w_{0}+w_{a} z /(1+z)$, is sensitive to the prior on the mean curvature of the universe (Linder 2005; Knox et al. 2006). The reason is that the response of the relative distance to a variation in $w_{a}$ resembles that to a variation in the mean curvature and that the SN technique lacks the calibration of absolute distances (Zhan \& Knox 2006). Even for a flat universe with $w(z) \equiv w_{0}$, the SN constraint on $w_{0}$ can be tightened considerably if the matter density is known to high precision (Frieman et al. 2003). Such priors may come from other techniques, such as the cosmic microwave background (CMB), weak lensing, and baryon acoustic oscillations (BAOs; Eisenstein et al. 1998; Cooray et al. 2001; Blake \& Glazebrook 2003; Hu \& Haiman 2003; Linder 2003; Seo \& Eisenstein 2003). It has indeed been demonstrated that the latter three techniques are highly complementary to the SN $D_{L}$ technique (Frieman et al. 2003; Seo \& Eisenstein 2003; Knox et al. 2006).

Since $\mathrm{SNe}$ explode in galaxies, their distribution bears the BAO imprint as well. To measure the $\mathrm{SN}$ spatial power spec-

\footnotetext{
${ }^{1}$ Department of Physics, University of California, Davis, CA 95616; hzhan@ucdavis.edu, tyson@physics.ucdavis.edu.

${ }^{2}$ Department of Physics, Texas A\&M University, College Station, TX 77843; wang@physics.tamu.edu.

${ }^{3}$ Department of Astronomy and Steward Observatory, University of Arizona, Tucson, AZ 85721; ppinto@as.arizona.edu.

${ }^{4}$ We consider only Type Ia SNe in this Letter.

${ }^{5}$ See http://www.lsst.org.
}

trum, one needs the angular position and redshift of each SN, not its luminosity. Hence, the SN BAO technique does not suffer from uncertainties in the SN standard candle, which constitute the largest unknown in the $D_{L}$ measurements. Nevertheless, the narrow range of the $\mathrm{SN}$ intrinsic luminosity reduces the effect of Malmquist-like biases and luminosity evolution. The SN rate traces the mass and star formation of the host galaxies with a time delay (Sullivan et al. 2006). This means that $\mathrm{SNe}$ have a different clustering bias than galaxies that are selected by their luminosity or color. Finally, SNe have rich and time-varying spectral features for accurate estimation of photometric redshifts (photo-zs) (Pinto et al. 2004; Wang 2007; Wang et al. 2007), which is helpful for measuring BAOs from a photometric survey.

There have been discussions of using the SN weak-lensing magnification (Cooray et al. 2006) and nearby SN peculiar velocities (Hannestad et al. 2007) to probe the large-scale structure. We focus on photometric SN surveys for BAOs in this Letter and note in passing that the SN weak-lensing technique is more limited by shot noise than the SN BAO technique (Zhan 2006; Zhan \& Knox 2006) and that the SN peculiar velocity technique requires precise redshift and distance measurements.

For the BAO technique to be useful, one must survey a large volume at a sufficient sampling density as uniformly as possible. Although SN events are rare, the spatial density of SNe accumulated over several years will be comparable to the densities targeted for future spectroscopic galaxy BAO surveys.

\section{PHOTOMETRIC SUPERNOVA SURVEYS}

We assume two photo- $z$ SN survey models: a shallow one (S20k) that covers 20,000 $\operatorname{deg}^{2}$ to $z=0.8$ for 10 years, and a deep one (D2k) that covers $2000 \mathrm{deg}^{2}$ to $z=1.2$ for 5 years. The S20k data may be extracted as one of the many products from the proposed LSST, and a dedicated ground-based SN survey will likely be sufficient to produce the D2k data. Since there is no precedent for such photo-z SN BAO surveys, we have to make crude estimates for the fiducial survey parameters and vary them to cover a wider range.

We calculate the observer-frame SN rate based on the restframe SN rates (Cappellaro et al. 1999; Hardin et al. 2000; Pain et al. 2002; Madgwick et al. 2003; Tonry et al. 2003; Blanc et al. 2004; Dahlen et al. 2004; Neill et al. 2006). The 
resulting number of SNe per steradian per unit redshift per year (observer frame) is roughly

$$
\begin{aligned}
\frac{d n}{d \Omega d z d t} \propto & {\left[\exp \left(3.12 z^{2.1}\right)-1\right] } \\
& \times \begin{cases}1, & z \leq 0.5, \\
\exp \left[-(z-0.5)^{2} / 2 a^{2}\right], & z>0.5,\end{cases}
\end{aligned}
$$

where the first term on the right-hand side fits the observed SN rate at $z \leq 0.55$, and the additional exponential term with $a=0.125(0.21)$ cuts off the distribution at $z \sim 0.8$ (1.2) for S20k (D2k). We take the efficiency with which S20k (D2k) will produce well-observed SN light curves suitable for accurate photo-zs from this distribution as $50 \%$ (100\%). The accumulated SN surface density is then $\Sigma=370$ and 980 $\mathrm{deg}^{-2}$ for S20k (7.4 million SNe in 10 years) and D2k (2 million $\mathrm{SNe}$ in 5 years), respectively.

We adopt a pedagogical convention and model the photo- $z$ error as a Gaussian with rms $\sigma_{z}=\sigma_{z 0}(1+z)$ and zero bias 6 $\delta z=0$. We assign for S20k $\sigma_{z 0}=0.02$ and for D2k $\sigma_{z 0}=$ 0.01 , which are achievable with simple photo- $z$ algorithms (Pinto et al. 2004; Wang et al. 2007).

Table 1 summarizes the SN surveys. It includes two additional quantities: the SN clustering bias $b$ and the cutoff wavenumber $k_{\max }$ for the BAO analysis, which is set to reduce the impact of nonlinear growth (see Eisenstein et al. [2007] for recovering high- $k$ information from spectroscopic surveys).

\section{FORECAST METHOD}

We use a modified forecast tool CSWAB (Zhan 2006) to assess the cosmological constraints from the clustering of SNe. In summary, the $\mathrm{SN}$ power spectrum in the $i$ th redshift bin $P_{i}\left(\boldsymbol{k}_{f}\right)$ reads (Seo \& Eisenstein 2003)

$$
\begin{aligned}
P_{i}\left(k_{f \perp}, k_{f \|}\right)= & \frac{D_{f, i}^{2} H_{i}}{D_{i}^{2} H_{f, i}}\left(1+\beta_{i} \mu^{2}\right)^{2} b_{i}^{2} G_{i}^{2} \mathcal{P}(k) \\
& \times \exp \left[-\left(c \sigma_{z, i} k_{\|} / H_{i}\right)^{2}\right]+s_{i},
\end{aligned}
$$

where the subscript $f$ denotes quantities in a reference cosmological model (the same as the fiducial model in the forecast), $D$ is the angular diameter distance, $H$ is the Hubble parameter, $\beta$ is the redshift distortion parameter, $\mu=k_{\|} / k, G$ is the linear growth factor, $\mathcal{P}(k)$ is the matter power spectrum at $z=0$, and $s=n^{-1}$ is the shot noise. The true wavenumbers $k_{\perp}$ and $k_{\|}$are related to the references by $k_{\perp}=k_{f \perp} D_{f} / D$ and $k_{\|}=k_{f \|} H / H_{f}$. Note that for a photo- $z$ survey with $\sigma_{z} \gtrsim 0.01(1+z)$, the radial BAO information is essentially lost, e.g., the power spectrum (excluding the shot noise) at the fundamental mode of BAOs $k_{\|} \sim 2 \pi / 150 \mathrm{Mpc}^{-1}$ is suppressed by a factor of 80 or more at $z=0.6$, although a photo- $z$ rms of $0.003(1+z)$ would recover that information.

CSWAB uses the Fisher matrix to estimate the error bounds of the parameters (Tegmark 1997):

$$
F_{\alpha \beta}^{\mathrm{BAO}}=\sum_{i} \frac{V_{f, i}}{2} \int \frac{\partial \ln P_{i}\left(\boldsymbol{k}_{f}\right)}{\partial q_{\alpha}} \frac{\partial \ln P_{i}\left(\boldsymbol{k}_{f}\right)}{\partial q_{\beta}} \frac{d \boldsymbol{k}_{f}}{(2 \pi)^{3}},
$$

where $V_{f}$ is the comoving survey volume and $\left\{q_{\alpha}\right\}$ is the pa-

\footnotetext{
${ }^{6}$ Any known photo- $z$ bias can be calibrated out in advance, so only the uncertainty of the bias matters.
}

TABLE 1

Supernova Survey Parameters

\begin{tabular}{ccccccc}
\hline \hline $\begin{array}{c}\text { Area } \\
\text { Survey }\end{array}$ & $\begin{array}{c}n \\
\left(\mathrm{deg}^{2}\right)\end{array}$ & $z$ & $\left(h^{3} \mathrm{Mpc}^{-3}\right)$ & $\sigma_{z}$ & $b$ & $\begin{array}{c}k_{\max } \\
\left(h \mathrm{Mpc}^{-1}\right)\end{array}$ \\
\hline S20k $\ldots . .$. & 20000 & 0.3 & $4.2 \times 10^{-4}$ & 0.026 & 1.18 & 0.17 \\
& & 0.5 & $6.3 \times 10^{-4}$ & 0.030 & 1.30 & 0.20 \\
& & 0.7 & $3.4 \times 10^{-4}$ & 0.034 & 1.42 & 0.24 \\
& \multirow{2}{*}{2000} & 0.3 & $4.2 \times 10^{-4}$ & 0.013 & 1.18 & 0.17 \\
& & 0.5 & $6.6 \times 10^{-4}$ & 0.015 & 1.30 & 0.20 \\
& & 0.7 & $7.8 \times 10^{-4}$ & 0.017 & 1.42 & 0.24 \\
& & 0.9 & $5.0 \times 10^{-4}$ & 0.019 & 1.54 & 0.29 \\
& & 1.1 & $1.8 \times 10^{-4}$ & 0.021 & 1.66 & 0.34 \\
\hline
\end{tabular}

Note. - The redshift is central to each bin, and the width of each bin is $\Delta z=0.2$

rameter set, which includes $b_{i}, \sigma_{z, i}, \delta z_{i}$, and $s_{i}$ of each bin and eight cosmological parameters: $w_{0}, w_{a}, \omega_{m}$ (the matter density), $\omega_{b}$ (the baryon density), $\theta_{s}$ (the angular size of the sound horizon at the last scattering surface), $\Omega_{k}$ (the curvature parameter), $n_{s}$ (the scalar spectral index), and $\Delta_{R}^{2}$ (the normalization of the primordial curvature power spectrum). The minimum marginalized error of $q_{\alpha}$ is $\sigma\left(q_{\alpha}\right)=\left(F^{-1}\right)_{\alpha \alpha}^{1 / 2}$. Independent Fisher matrices are additive; a prior on $q_{\alpha}, \sigma_{P}\left(q_{\alpha}\right)$, can be introduced via $F_{\alpha \alpha}^{\text {new }}=F_{\alpha \alpha}+\sigma_{P}^{-2}\left(q_{\alpha}\right)$. The fiducial values of $b_{i}, \sigma_{z, i}$, and $s_{i}^{-1}$ are listed in Table $1, \delta z_{i}=0$, and $\left(w_{0}, w_{a}, \omega_{m}, \omega_{b}, \theta_{s}, \Omega_{k}, n_{s}\right.$, $\left.\Delta_{R}^{2}\right)=\left(-1,0,0.127,0.0223,0.596^{\circ}, 0,0.951,2.0 \times 10^{-9}\right)$ from the WMAP 3 year results (Spergel et al. 2007). Unless stated otherwise, we always include fairly weak priors $\sigma_{P}\left(\ln b_{i}\right)=0.3, \sigma_{P}\left(\ln \Delta_{R}^{2}\right)=0.2, \sigma_{P}\left(\ln \theta_{s}\right)=\sigma_{P}\left(n_{s}\right)=0.05$, and $\sigma_{P}\left(\delta z_{i}\right)=2^{-1 / 2} \sigma_{P}\left(\sigma_{z, i}\right)=0.25 \sigma_{z, i}$.

To show the complementarity between the SN BAO and $D_{L}$ techniques, we include the $D_{L}$ constraints at the end of $\S 4$. The Fisher matrix for $\mathrm{SN} D_{L}$ is

$$
\begin{gathered}
F_{\alpha \beta}^{D_{L}}=\frac{1}{\sigma_{m}^{2}} \int n_{p}\left(z_{p}\right) \frac{\partial \bar{m}_{p}\left(z_{p}\right)}{\partial q_{\alpha}} \frac{\partial \bar{m}_{p}\left(z_{p}\right)}{\partial q_{\beta}} d z_{p}, \\
\bar{m}_{p}=\int\left[5 \log D_{L}(z)+M+e_{1} z+e_{2} z^{2}\right] p\left(z \mid z_{p}\right) d z,
\end{gathered}
$$

where $\sigma_{m}=0.15$ is the scatter of the SN apparent magnitude, the subscript $p$ signifies photo- $z$ space, $M$ is the $\mathrm{SN}$ absolute magnitude, $e_{1}$ and $e_{2}$ account for possible SN evolution, and $p\left(z \mid z_{p}\right)$ is the probability density of a $\mathrm{SN}$ at $z$ given its photo$z z_{p}$. Following Albrecht et al. (2006) we impose a prior of 0.015 on $e_{1}$ and $e_{2}$ and let $M$ float. We also include $z<0.2 \mathrm{SNe}$ in $F^{D_{L}}$, because they are important for cosmology with $D_{L}$ (Linder 2006).

\section{COSMOLOGICAL CONSTRAINTS}

The marginalized $1 \sigma$ errors of a subset of the cosmological parameters are given in Table 2. The two photo- $z$ SN BAO surveys place rather weak constraints on the dark energy EOS even with Planck priors. Nevertheless, they provide moderate constraints on the matter density $\omega_{m}$, baryon density $\omega_{b}$, and curvature parameter $\Omega_{k}$, which are helpful for the SN $D_{L}$ technique. The smaller area of $\mathrm{D} 2 \mathrm{k}$ is compensated by its greater depth and better photo-zs, so that D2k performs nearly as well as S20k. Spectroscopic BAO surveys of similar characteristics can reduce the error on $\omega_{m}, \omega_{b}$, and $\Omega_{k}$ by a factor of 2 and significantly more on $w_{0}$ and $w_{a}$.

The baryon signature has been detected at $\sim 3 \sigma$ level [and $\sigma\left(\ln \omega_{m}\right) \sim 10 \%$ ] from SDSS luminous red galaxies, both spec- 
TABLE 2

Marginalized $1 \sigma$ Errors on Selected Cosmological Parameters FROM SN BAOS

\begin{tabular}{|c|c|c|c|c|c|c|}
\hline Surveys & $w_{0}$ & $w_{a}$ & $w_{p}$ & $\begin{array}{c}\ln \omega_{m} \\
\left(\times 10^{-2}\right)\end{array}$ & $\begin{array}{c}\ln \omega_{b} \\
\left(\times 10^{-2}\right)\end{array}$ & $\begin{array}{r}\Omega_{k} \\
\left(\times 10^{-2}\right)\end{array}$ \\
\hline S20k & 0.85 & 2.9 & 0.22 & 23 & 38 & 1.8 \\
\hline $\mathrm{S} 20 \mathrm{k}+$ Planck & 0.66 & 1.8 & 0.12 & 0.83 & 0.91 & 0.55 \\
\hline $\mathrm{D} 2 \mathrm{k}$ & 0.96 & 3.0 & 0.19 & 25 & 42 & 2.2 \\
\hline $\mathrm{D} 2 \mathrm{k}+$ Planck ....... & 0.74 & 2.0 & 0.13 & 0.85 & 0.91 & 0.60 \\
\hline
\end{tabular}

NotES. - The error of the pivot EOS $w_{p}$ equals that of a constant EOS, and the Dark Energy Task Force figure of merit (Albrecht et al. 2006) equals $0.052\left[\sigma\left(w_{a}\right) \times \sigma\left(w_{p}\right)\right]^{-1}$.

troscopically (Eisenstein et al. 2005) and photometrically (Blake et al. 2007; Padmanabhan et al. 2007). These detections assume a flat universe with a cosmological constant and a fixed scalar spectral index $n_{s}$. Under the same assumptions, S20k BAO can constrain $\omega_{m}$ to $8 \%$ and $\omega_{b}$ to $15 \%$. If $\omega_{b}$ is fixed as well (as in Eisenstein et al. 2005), S20k BAO can achieve $\sigma\left(\ln \omega_{m}\right)=1.5 \%$.

Theoretical uncertainties in the redshift distortion, nonlinear evolution, and scale-dependent clustering bias can be important to BAOs (Seo \& Eisenstein 2005; White 2005; Guzik et al. 2007). For a simple test, we replace the linear redshift distortion factor $\left(1+\beta \mu^{2}\right)^{2}$ (Kaiser 1987) in equation (1) with $1+$ $2 e \beta \mu^{2}+f \beta^{2} \mu^{4}$, where $e=f=1$ are parameters accounting for our uncertain knowledge of the redshift distortion (Scoccimarro 2004). We take priors $\sigma_{P}(e)=0.05$ and $\sigma_{P}(f)=0.1$ and find a less than $1 \%$ change to the results in Table 2. Spectroscopic (galaxy) surveys with the same redshift distribution and coverage will see $w_{0}$ and $w_{a}$ errors doubled in this test, because they have more information to lose.

Figure 1 explores the dependence of the $\mathrm{SN}$ BAO error product $\sigma\left(w_{a}\right) \times \sigma\left(w_{p}\right)(\mathrm{EP})$ on the photo- $z$ rms $\sigma_{z}$ and prior $\sigma_{P}\left(\sigma_{z}\right)$. At a large $\sigma_{z}$, there is little radial BAO information, so that the Fisher matrix $F^{\mathrm{BAO}}$ scales roughly with the $k$-space volume, which is proportional to $H / c \sigma_{z}$. This leads to a scaling $\mathrm{EP} \propto \sigma_{z}$ when $\sigma_{z}$ and $\delta z$ are known accurately (dashed lines), consistent with Seo \& Eisenstein (2003) and Zhan (2006). When $\sigma_{z} \lessgtr 0.008(1+z)$, the radial BAOs become available, and, hence, the EP slope steepens at smaller $\sigma_{z}$.

Figure 2 shows the EP contours (solid lines) of the shallow survey as the projected SN number density and survey area vary. Overplotted in dotted lines are contours of the total number of SNe. If the number of SNe is taken as a crude proxy for effort, one can optimize the survey (in terms of dark energy constraints) by searching the minimum EP on the constanteffort curve. This means that for $\Sigma \gtrsim 200 \mathrm{deg}^{-2}$ and area less than $30,000 \mathrm{deg}^{2}$, one would always choose the maximum survey area possible for SN BAO as opposed to accumulating more $\mathrm{SNe}$ in a smaller area.

Finally, we illustrate the complementarity between the SN BAO and $D_{L}$ techniques in Figure 3. Although the S20k BAO technique (dashed line) does not place useful constraints on dark energy, its combination (shaded area) with the $D_{L}$ technique (dotted line) reduces the EP by a factor of 5.5 over the $D_{L}$-alone EP. Moreover, the combined result (solid line) is not very sensitive to the $\mathrm{CMB}$ priors, because the $\mathrm{BAO}$ technique can provide adequate constraints on cosmological parameters, such as $\omega_{m}$ and $\Omega_{k}$, for the $D_{L}$ technique.

\section{DISCUSSION AND CONCLUSIONS}

We have demonstrated that photo- $z \mathrm{SN}$ data can be used to measure $\mathrm{BAOs}$ and to constrain cosmological parameters. The

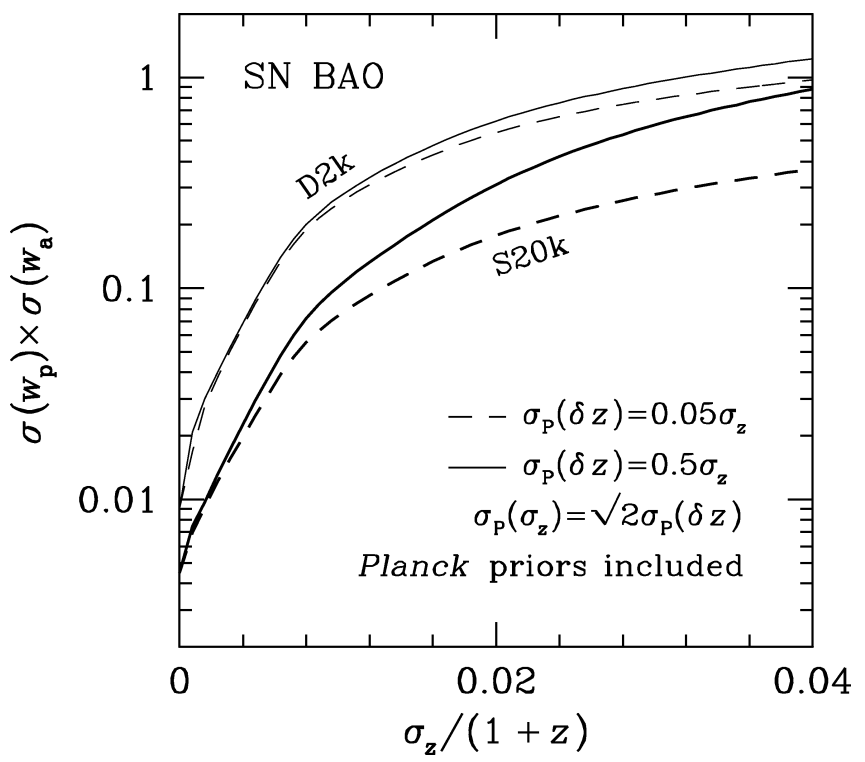

FIG. 1.- EP $\sigma\left(w_{p}\right) \times \sigma\left(w_{a}\right)$ of S20k (thick lines) and D2k (thin lines) as a function of the rms photo- $z$ error $\sigma_{z}$. The priors on the photo- $z$ biases are taken to be $0.5 \sigma_{z}$ (solid lines) and $0.05 \sigma_{z}$ (dashed lines), which correspond to calibrations with 4 and 400 spectra per redshift bin, respectively, in the Gaussian case. To reduce the dimensions, we peg the prior on the photo- $z$ rms to that on the photo- $z$ bias: $\sigma_{P}\left(\sigma_{z}\right)=\sqrt{2} \sigma_{P}(\delta z)$. For comparison, LSST weak lensing, galaxy BAOs, and the two combined will achieve $\mathrm{EP} \sim 0.01,0.02$, and 0.002 , respectively (Zhan 2006).

BAO constraints on the matter density and the baryon density are sensitive to the priors on the curvature and the scalar spectral index but not to the dark energy parameters. The dark energy constraints from the SN BAO technique alone are not meaningful. However, a combination of the SN BAO and $D_{L}$ techniques reduces the EP considerably, and the Planck priors

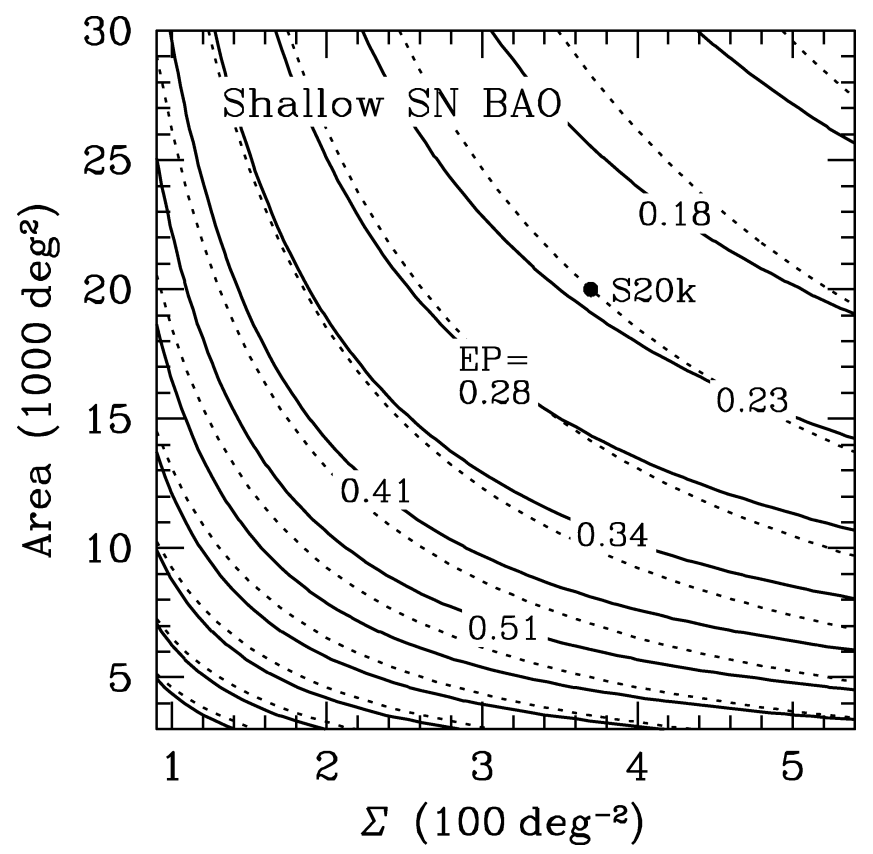

FIG. 2.-EP $\sigma\left(w_{p}\right) \times \sigma\left(w_{a}\right)$ (solid lines) as a function of the survey area and SN surface density $\Sigma$ (accumulated over 10 years). The EP contours are spaced logarithmically. The SN distribution is scaled from S20k (filled circle; 7.4 million SNe) by $\Sigma$. Photo- $z$ parameters are the same as those of S20k, and the Planck priors are assumed. The total number of $\mathrm{SNe}$ is held constant along each dotted line, which is spaced at factors of $\sqrt{2}$. 


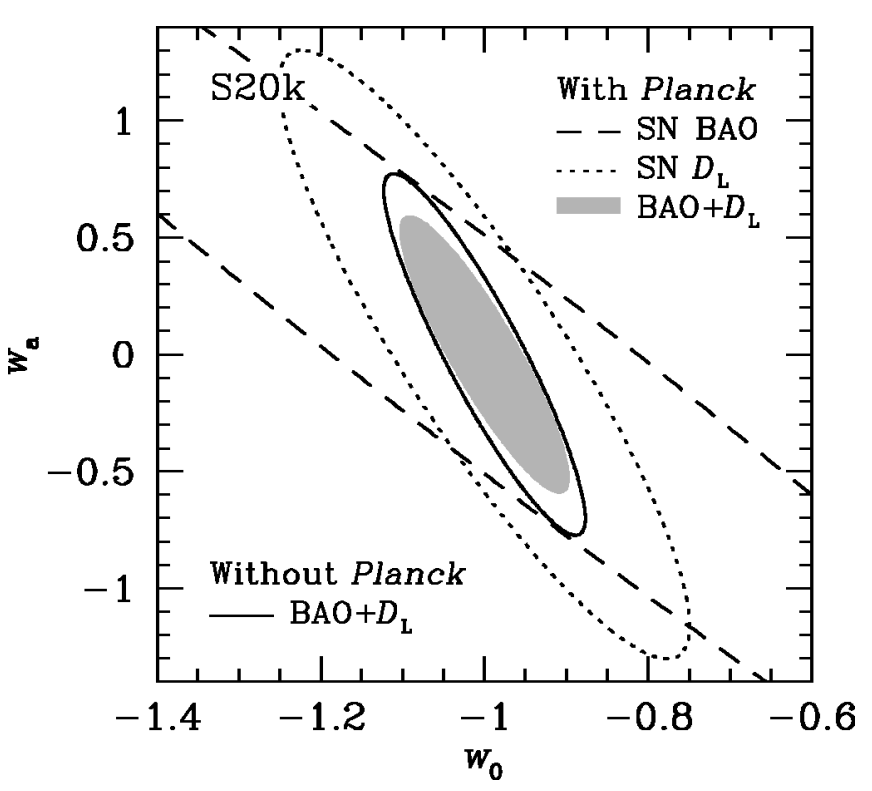

FIG. 3.-Marginalized $1 \sigma$ error contours of the dark energy EOS parameters $w_{0}$ and $w_{a}$ from S20k SN BAOs (dashed line; $\mathrm{EP}=0.22$ with Planck), luminosity distances (dotted line; EP $=0.064$ with Planck), and the two combined with (shaded area; $\mathrm{EP}=0.012$ ) and without (solid line; $\mathrm{EP}=0.018$ ) Planck. are no longer crucial. The SN BAO results in $\S 4$ are also applicable to photo- $z$ galaxy BAOs. We note that photo- $z$ errors are a large uncertainty for photo- $z$ SN cosmology. Although our assumption about them is conservative compared to the results in Pinto et al. (2004) further studies are needed to make realistic forecasts.

Long and nonuniform cadence may result in uneven sampling of the SN spatial distribution. Fortunately, LSST will be likely to always catch the $\mathrm{SNe}$ (especially high- $z$ ones) at their maximum owing to its fast sky coverage and rapid sampling. Furthermore, the effect of the cadence on the SN depth can be simulated and determined, and methods of correcting for uneven depths in galaxy surveys can be applied to the SN data.

A spectroscopic SN BAO survey will be impractical, because one would have to revisit the sky many times spectroscopically over thousands of square degrees to catch the SNe that occur at different times. However, LSST will be able to obtain SNe in the millions over half the sky photometrically. This opens a window for applying the BAO technique to SNe and achieving more robust constraints with photo- $z$ SN data. Since this SN BAO analysis requires no additional observations beyond doing the SN $D_{L}$ analysis alone, it should be a feature of all large-area SN cosmology analyses. Moreover, the SN data can also help calibrate the host galaxy photo-zs and the photo- $z$ error distribution of other galaxies through the cross-correlation method (Schneider et al. 2006; Zhan 2006; Newman 2008).

We thank J. Frieman, L. Knox, and M. Wood-Vasey for useful conversations. This work was partially supported by a UC Davis Academic Federation Innovative Developmental Award.

\section{REFERENCES}

Albrecht, A., et al. 2006, preprint (astro-ph/0609591)

Astier, P., et al. 2006, A\&A, 447, 31

Blake, C., Collister, A., Bridle, S., \& Lahav, O. 2007, MNRAS, 374, 1527

Blake, C., \& Glazebrook, K. 2003, ApJ, 594, 665

Blanc, G., et al. 2004, A\&A, 423, 881

Cappellaro, E., Evans, R., \& Turatto, M. 1999, A\&A, 351, 459

Cooray, A., Holz, D. E., \& Huterer, D. 2006, ApJ, 637, L77

Cooray, A., Hu, W., Huterer, D., \& Joffre, M. 2001, ApJ, 557, L7

Dahlen, T., et al. 2004, ApJ, 613, 189

Eisenstein, D. J., Hu, W., \& Tegmark, M. 1998, ApJ, 504, L57

Eisenstein, D. J., Seo, H.-J., Sirko, E., \& Spergel, D. N. 2007, ApJ, 664, 675

Eisenstein, D. J., et al. 2005, ApJ, 633, 560

Frieman, J. A., Huterer, D., Linder, E. V., \& Turner, M. S. 2003, Phys. Rev. $\mathrm{D}, 67,083505$

Frieman, J. A., et al. 2008, AJ, 135, 338

Guzik, J., Bernstein, G., \& Smith, R. E. 2007, MNRAS, 375, 1329

Hannestad, S., Haugboelle, T., \& Thomsen, B. 2007, J. Cosmol. Astropart. Phys., submitted (astro-ph/0705.0979)

Hardin, D., et al. 2000, A\&A, 362, 419

Hu, W., \& Haiman, Z. 2003, Phys. Rev. D, 68, 063004

Kaiser, N. 1987, MNRAS, 227, 1

Knox, L., Song, Y.-S., \& Zhan, H. 2006, ApJ, 652, 857

Linder, E. V. 2003, Phys. Rev. D, 68, 083504

2005, Astropart. Phys., 24, 391

2006, Phys. Rev. D, 74, 103518
Madgwick, D. S., Hewett, P. C., Mortlock, D. J., \& Wang, L. 2003, ApJ, 599, L33

Miknaitis, G., et al. 2007, ApJ, 666, 674

Neill, J. D., et al. 2006, AJ, 132, 1126

Newman, J. 2008, ApJ, submitted

Padmanabhan, N., et al. 2007, MNRAS, 378, 852

Pain, R., et al. 2002, ApJ, 577, 120

Perlmutter, S., et al. 1999, ApJ, 517, 565

Phillips, M. M. 1993, ApJ, 413, L105

Pinto, P. A., Smith, C. R., \& Garnavich, P. M. 2004, BAAS, 36, 1530

Riess, A. G., et al. 1998, AJ, 116, 1009

Schneider, M., Knox, L., Zhan, H., \& Connolly, A. 2006, ApJ, 651, 14

Scoccimarro, R. 2004, Phys. Rev. D, 70, 083007

Seo, H., \& Eisenstein, D. J. 2003, ApJ, 598, 720

Seo, H.-J., \& Eisenstein, D. J. 2005, ApJ, 633, 575

Spergel, D. N., et al. 2007, ApJS, 170, 377

Sullivan, M., et al. 2006, ApJ, 648, 868

Tegmark, M. 1997, Phys. Rev. Lett., 79, 3806

Tonry, J. L., et al. 2003, ApJ, 594, 1

Tyson, J. A. 2006, in AIP Conf. Proc. 870, Intersections of Particle and Nuclear Physics, ed. T. M. Liss (New York: AIP), 44

Wang, Y. 2007, ApJ, 654, L123

Wang, Y., Narayan, G., \& Wood-Vasey, M. 2007, MNRAS, 382, 377

White, M. 2005, Astropart. Phys., 24, 334

Zhan, H. 2006, J. Cosmol. Astropart. Phys., 8, 8

Zhan, H., \& Knox, L. 2006, preprint (astro-ph/0611159) 